\title{
Nipping it in the bud: The role of primary care in early prevention and management of childhood obesity
}

\author{
Denise Darmawikarta
}

\author{
University of Toronto
}

The prevalence of obesity in Canadian children has more than doubled in the last few decades, putting Canada in the midst of a childhood obesity epidemic. ${ }^{1}$ Unfortunately, obesity in childhood is often carried into adulthood and can become a significant risk factor for common chronic diseases including hypertension, coronary heart disease and several forms of cancer. ${ }^{1}$ Perhaps more alarming is the consequent emergence of type 2 diabetes (T2D), previously labeled an adult disease, in the younger age groups; in fact, nearly all children who are diagnosed with T2D are overweight or obese. ${ }^{2}$ Given the many downstream comorbidities associated with childhood obesity, efforts to prevent and manage it are of utmost importance.

Not surprisingly, although some individuals have a genetic predisposition to obesity, the causes are not only biological, but also social. ${ }^{1}$ Sociological factors, such as ethnicity, socioeconomic status, and area of residence, play important roles in determining the risk of obesity. ${ }^{1}$ For instance, a higher prevalence of obesity is observed in aboriginals compared to non-aboriginals. ${ }^{1}$ Thus, it is prudent to reason that the success of any obesity prevention and management strategy is dependent on its ability to address both intrinsic patient characteristics and external environmental factors that affect the disease risk.

Given the frequent contact between a family physician, pediatrician or nurse practitioner and parents with children, primary care has emerged as a promising avenue for the development of preventative care and early intervention strategies. ${ }^{3}$ Primary care providers have a unique opportunity to address childhood obesity because they have longitudinal access to patient histories that will assist in the early identification of at-risk individuals, which often yield greater success in subsequent treatment. ${ }^{4}$ In turn, physicians who are successful in referring their patients to effective treatment programs will positively reinforce their screening behaviours. ${ }^{4}$ One can also imagine that primary care providers are more likely to be equipped to develop rapport and encourage compliance in their patients through a holistic understanding of the different cultural values and socioeconomic status within the region they serve. Currently, however, studies show that primary care providers lack the knowledge and skills to assess and manage childhood obesity. ${ }^{5}$ What this necessitates is further research to facilitate the development of systematic, evidence-based primary care strategies to guide physicians through the diagnosis, treatment and prevention of obesity in children.

Practice based research networks (PBRNs) may provide a much-needed solution. PBRNs are groups of healthcare providers or medical clinics typically situated in nonacademic environments that are networked for the purposes of evaluating and advancing healthcare practices in communities, including improving disease screening and prevention strategies. ${ }^{3}$ Although standardized tools for assessing the effectiveness of PBRNs are yet to be established, ${ }^{6}$ PBRNs can accomplish many benefits for the medical field, policy-makers and the public in the battle against childhood obesity. They enable family physicians to be principal investigators, thereby allowing them to contribute to cutting-edge research, to practice, learn and reflect in order to improve patient care. ${ }^{7}$ They also promote collaboration between primary care providers and researchers, allowing for the continual evolution of study protocols that arise from experiences as front-line care providers. ${ }^{7}$ In addition, they can encourage children, families and other members of the community (teachers, counselors, etc.) to be active participants in achieving the goal of building healthier communities, a feature often not available to traditional academic centre-based research. ${ }^{3}$ Ultimately, PBRNs serve to assemble an evidence base for the development of pragmatic, primary care prevention and management strategies that community healthcare 
providers themselves can incorporate into their everyday practice. More simply, PBRNs are the translational bridge that fills the gap between bench and bedside.

Recently, the Public Health Agency of Canada, in partnership with the College of Family Physicians of Canada and the Canadian Institute for Health Information, has committed to a 5-year contribution agreement to continue the development of the Canadian Primary Care Sentinel Surveillance Network (CPCSSN) ${ }^{8}$ CPCSSN was founded to enable the monitoring of chronic diseases and serves as a resource for primary care research; ${ }^{8}$ it represents the first step towards improving primary care practices across Canada. Unfortunately, local PBRNs, such as TARGetKids!, 9 a Toronto-based pediatric PBRN specifically addressing childhood obesity, must still rely on funding from external sources or operating grants from the Canadian Institutes of Health Research, ${ }^{10}$ which is unsustainable over the long term.

Given the potential for PBRNs to truly improve the health status of populations, we need to seriously consider them as frontrunners in the race towards childhood obesity reduction. Our efforts need to be focused on cultivating these "laboratories of primary care", 7 especially since the majority of clinical and preventative care is delivered in these settings. If we are determined to build healthier Canadians, we must begin early and nip obesity in its bud.

\section{References}

1. Public Health Agency of Canada and the Canadian Institute for Health Information. Obesity in Canada. 2011.

2. Fagot-Campagna A, Pettitt DJ, Engelgau MM, Burrows NR, Geiss LS, Valdez $R$, et al. Type 2 diabetes among North American children and adolescents: an epidemiologic review and a public health perspective. J Pediatr 2000 May;136(5):664-672.

3. Tapp $\mathrm{H}$, Dulin $\mathrm{M}$. The science of primary health-care improvement: potential and use of community-based participatory research by practicebased research networks for translation of research into practice. Exp Biol Med (Maywood) 2010 Mar;235(3):290-299.

4. Haemer M, Cluett S, Hassink SG, Liu L, Mangarelli C, Peterson T, et al. Building capacity for childhood obesity prevention and treatment in the medical community: call to action. Pediatrics 2011 Sep;128 Suppl 2:S71-7.

5. van Gerwen M, Franc C, Rosman S, Le Vaillant M, Pelletier-Fleury N. Primary care physicians' knowledge, attitudes, beliefs and practices regarding childhood obesity: a systematic review. Obes Rev 2009 Mar;10(2):227-236.

6. Bleeker JM, Stalman WA, van der Horst HE. Evaluating primary care research networks: a review of currently available tools. J Am Board Fam Med 2010 Jul-Aug;23(4):465-475.

7. Jones $C$. Laboratories of primary care: practice-based research networks in Canada. Can Fam Physician 2006 Sep;52(9):1045-6, 1047-8.

8. Birtwhistle RV. Canadian Primary Care Sentinel Surveillance Network: a developing resource for family medicine and public health. Can Fam Physician 2011 Oct;57(10):1219-1220.

9. HISTORY: High Impact Strategies Towards Overweight Reduction in Youth. Available at: http://obesityinyouth.org/home/, 2011.

10. Grzybowski S, Wallace L. Primary care research in Canada and the United States. Ann Fam Med 2006 Sep-Oct;4(5):466-467.

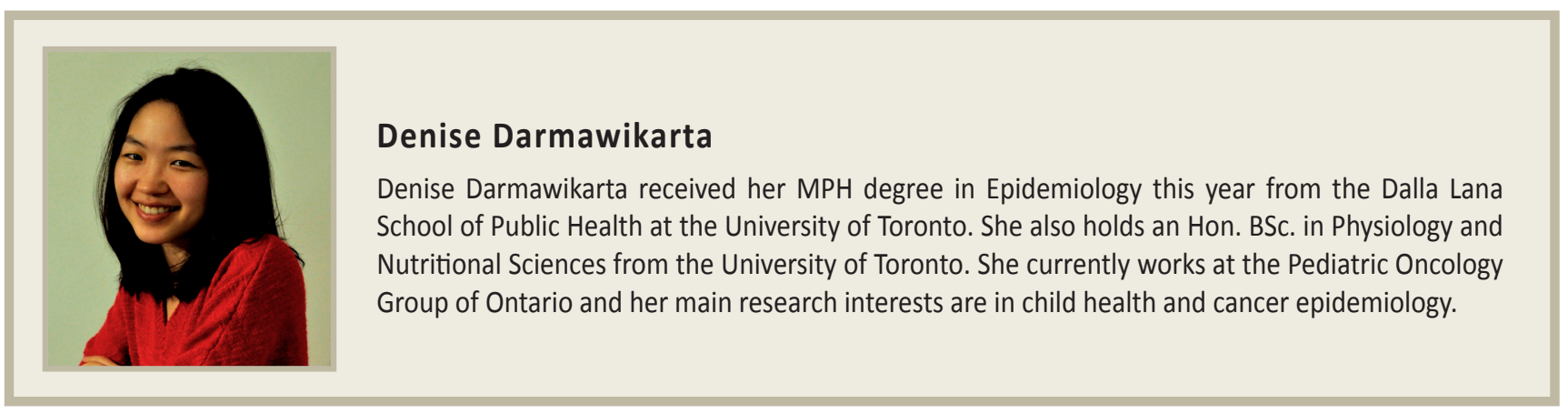

